\title{
A design-constraint trade-off underpins the diversity in ecologically important traits in species Escherichia coli
}

\author{
Katherine Phan and Thomas Ferenci \\ School of Molecular Bioscience, University of Sydney, Sydney, New South Wales, Australia
}

\begin{abstract}
Bacterial species are internally diverse in genomic and multi-locus gene comparisons. The ecological causes of phenotypic and genotypic diversity within species are far less well understood. Here, we focus on the competitive fitness for growth on nutrients within Escherichia coli, an internally rich species. Competition experiments in nutrient-limited chemostats revealed that members of the ECOR collection exhibited a wide continuum of competitive abilities, with some fitter and some less fit than the lab strain MG1655. We observed an inverse relationship between competitiveness and the resistance of strains to detergent and antibiotic, consistent with the notion that membrane permeability and competitive fitness are linked by a trade-off between selfpreservation and nutritional competence (SPANC); high permeability has a postulated cost in antibacterial sensitivity whereas a low permeability has a cost in nutrient affinity. Isolates moved along the markedly nonlinear trade-off curve by mutational adaptation; an ECOR strain sensitive to antibacterials and a good competitor was easily converted by mutation into a mutant with higher resistance but poorer competition in the presence of low antibiotic concentrations. Conversely, a resistant ECOR strain changed into a better competitor after a short period of selection under nutrient limitation. In both directions, mutations can affect porin proteins and outer membrane permeability, as indicated by protein analysis, gene sequencing and an independent assay of outer membrane permeability. The extensive, species-wide diversity of $E$. coli in ecologically important traits can thus be explained as an evolutionary consequence of a SPANC trade-off driven by antagonistic pleiotropy.
\end{abstract}

The ISME Journal (2013) 7, 2034-2043; doi:10.1038/ismej.2013.82; published online 16 May 2013

Subject Category: Microbial ecology and functional diversity of natural habitats

Keywords: bacterial diversity; trade-offs; nutrient uptake; outer membranes; antibiotic susceptibility

\section{Introduction}

The costs and benefits of adaptational change provide a recurring theme in all organisms studied in different environments (Stearns, 1989; Roff and Fairbairn, 2007). Most such studies have focused on trade-offs in plants and animals, whereas the operation of trade-offs has been much less systematically studied with microorganisms. This is unfortunate because in the examples using bacteria and phages, it is clear that microbes are much more amenable to the analysis of not just the effects but also the potential molecular causes of trade-offs (De Paepe and Taddei, 2006; Jessup and Bohannan, 2008; Mroczkowska and Barlow, 2008; Tree et al., 2008; Lang et al., 2009; Lee et al., 2009; Kang and Park, 2010; De Paepe et al., 2011; Taylor and Buckling, 2011). Here, we identify mutually

Correspondence: T Ferenci, School of Molecular Bioscience, University of Sydney, Sydney 2006, New South Wales, Australia. E-mail: tom.ferenci@sydney.edu.au

Received 30 October 2012; revised 10 April 2013; accepted 17 April 2013; published online 16 May 2013 exclusive traits in Escherichia coli to define an ecological trade-off between self-protection and growth affinity in species-wide terms as well as in molecular detail.

A genetic explanation of fitness trade-offs between environments is the principle of antagonistic pleiotropy, in which mutations or traits that are beneficial in one environment are harmful in others (Levins, 1968). Antagonistic pleiotropy is evident amongst mutations selected during experimental evolution of bacteria in different environments (Cooper and Lenski, 2000; MacLean et al., 2004); individual mutations with these effects are beginning to be identified (King et al., 2004; Maharjan et al., 2013). For example, a mutation resulting in antagonistic pleiotropy can alter the trade-off between self-preservation and nutritional competence (SPANC) balance (Ferenci, 2005). Mutations in $r p o S$, the gene regulating the general stress response (Hengge, 2011), result in an altered resource allocation of transcriptional space between stress genes and vegetative growth genes; bacteria that express high levels of resistance divert resources from vegetative genes and are thus less competitive in 
nutrient-limited environments (Notley-McRobb et al., 2002). Mutations in central regulation or rpoS itself can change this resource allocation, and hence change the resistance phenotype (Ferenci et al., 2011). This flexible resource allocation at the level of RNA polymerase provides perhaps the best understood example of a trade-off with a molecular explanation (Nystrom, 2004; Ferenci, 2005; Gudelj et al, 2010).

Although resource allocation problems are common to many organisms (Agrawal et al., 2010), alternative mechanisms for a stress-resistance-multiplication trade-off can be envisaged. In the example shown in Figure 1, a design constraint on mutually exclusive cellular characteristics is the source of a possible trade-off; the design constraint is that the outer membrane of bacteria is evolved to exclude toxic compounds but at the same time needs to provide access to nutrients. The barrier function of the outer membrane is sophisticated (Nikaido, 2003) but compromised by the need to permit diffusible access to nutrients through channel-forming proteins called porins. The general permeability into E. coli of various structurally diverse polar nutrients and antibiotics is determined by the type of porin protein expressed in cells (Liu and Ferenci, 2001; Nikaido, 2003; Pages et al., 2008; Delcour, 2009). The major porins of E. coli, $\mathrm{OmpF}$ and $\mathrm{OmpC}$, form relatively nonspecific water-filled channels but act as molecular sieves; the difference between them is that the OmpC channels are more restrictive (Liu and Ferenci, 2001; Nikaido, 2003; Pages et al., 2008; Delcour, 2009). Several previous studies have shown that bacteria containing only OmpC are more resistant to toxic hydrophilic agents than those containing OmpF. Indeed, in clinical isolates, a contributing factor to drug exclusion is the mutational loss of OmpF or in more extreme cases, both OmpF and OmpC (Pages et al., 2008). In addition,

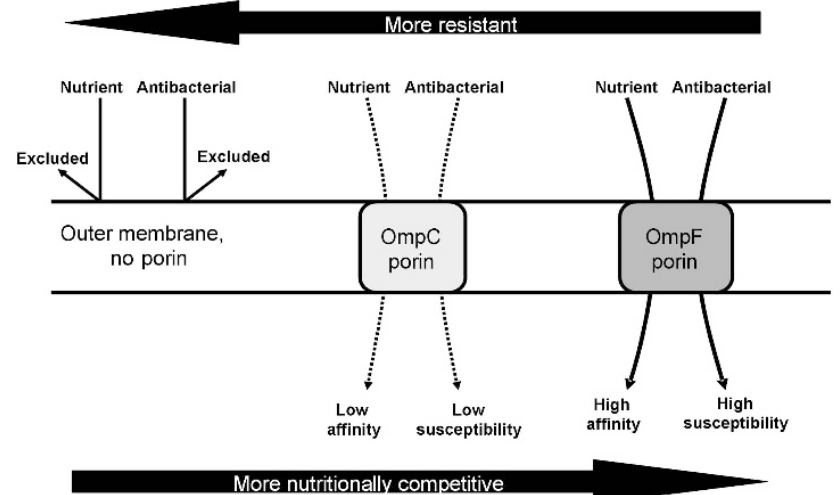

Figure 1 Membrane permeability effects with the major porins of E. coli. The outer membrane excludes polar nutrients and antibacterial compounds, whereas OmpC porin with limited permeability provides lower general permeability than OmpF, which is the most open porin in E. coli. The schematic is based on data discussed in Liu and Ferenci (2001); Nikaido (2003); Pages et al. (2008); Delcour (2009)). bile-salt (detergent) resistance in E. coli is readily altered by changes of porin regulation in the intestinal environment (De Paepe et al., 2011). The fitness cost of porin changes has not been evaluated systematically, although completely porinless mutants exhibit a significant growth defect on most substrates (Bavoil et al., 1977). In this study, we show that restricting the quantity or pore size of porins, as occurs naturally in some E. coli isolates, has a large deleterious effect on competition for nutrients.

These findings and Figure 1 all point to a resistance-nutrition trade-off involving porins, and here we systematically investigate the influence of this trade-off on the diversity and fitness of members of a species. E. coli shows considerable genomic diversity (Touchon et al., 2009) and we used the taxonomically well-studied ECOR collection (Ochman and Selander, 1984; Selander et al., 1987) that contains strains from numerous geographical sources and animal types. Here, we report on the extensive variation in competitive fitness and sensitivity to antibacterials within the species E. coli and the trade-off linking these properties.

Indeed, the most important ecological and evolutionary consequence of trade-offs is the enhanced possibility of coexistence and diversification (Levins, 1968; Gudelj et al., 2010). Constant mutational selection in fluctuating environments or environments where neither of two traits is entirely beneficial can lead to the mutational reassortment of the SPANC balance. In the case of the rpoSdetermined trade-off, there is selection for intermediate settings of the SPANC balance when bacteria are evolving under nutrient limitation but moderate environmental stress (King et al., 2006). This propensity for optimizing fitness in distinct environments leads to species-wide diversity in the concentration of RpoS (and molecules that regulate RpoS) across isolates of $E$. coli in the same environment (Ferenci et al., 2011). Here we demonstrate that other protective mechanisms, such as the outer membrane barrier in Gram-negative bacteria, are also highly diverse within a species, consistent with the notion that competitive fitness and antibiotic susceptibility are traits that often reassort along a nonlinear trade-off curve and contribute to intraspecies diversity.

\section{Materials and methods}

Bacterial strains

The 72 ECOR isolates (Ochman and Selander, 1984) were from a stock held and studied by Peter Reeves (Pupo et al., 2000). BW3779 (MG1655 otsA::Tn10) was constructed by $\mathrm{P} 1$ phage transduction of the otsA::Tn10 from UE60 (Giaever et al., 1988) into MG1655. ECOR5E was derived from ECOR5 after 10 days in a chemostat at a growth rate of $0.1 \mathrm{~h}^{-1}$ (34 generations) fed with $0.1 \times$ M9 (Miller, 1972) 
supplemented with $4 \%$ (vol/vol) low-salt LuriaBertani (LSLB; $10 \mathrm{gl}^{-1}$ tryptone, $5 \mathrm{~g} \mathrm{l}^{-1}$ yeast extract). ECOR59E was derived as a resistant mutant from ECOR59 on a $1-\mu \mathrm{g} \mathrm{ml}^{-1}$ chloramphenicol (Cml) plate.

\section{Competitive fitness assay}

For fitness measurements, ECOR strains were com peted against the reference K-12 BW3779 in chemostats. The competitions were with either limiting rich medium or limiting sugars. The chemostats were fed with either $0.1 \times$ M9 supplemented with $4 \%$ (vol/vol) LSLB or $0.1 \times$ M9 supplemented with $0.005 \%$ (wt/vol) glucose, $0.005 \%$ (wt/vol) galactose, $0.005 \%$ (wt/vol) fructose and $0.005 \%$ (wt/vol) xylose. The BW3779 and competing ECOR strain were grown in independent chemostats at $37^{\circ} \mathrm{C}$ overnight at $0.1 \mathrm{~h}^{-1}$ dilution rate. Cultures were mixed 50:50 in the same medium to start competition at the same dilution rate. The progress of the competition was monitored from samples taken at 1 , 24 and $48 \mathrm{~h}$. Bacteria in the mixed population were counted by serial dilution and plating onto Luria (L) agar (for total counts) with or without $15 \mu \mathrm{g} \mathrm{ml}^{-1}$ tetracycline plates. Strains were differentiated using tetracycline resistance (encoded in the neutral otsA::Tn10 marker (Maharjan et al., 2006)).

\section{Antibacterial susceptibility assays}

Sensitivity to $\mathrm{Cml}$ or SDS was assayed from midexponential cultures grown in LSLB. Bacterial density was adjusted to a standard value (optical density at $600 \mathrm{~nm} 0.5)$ before spotting cultures $(2 \mu \mathrm{l})$ onto LSL-agar plates or LSL-agar containing either $0.1 \%$ (wt/vol) SDS or $3 \mu \mathrm{g} \mathrm{ml}^{-1} \mathrm{Cml}$. After $15 \mathrm{~h}$ growth at $37^{\circ} \mathrm{C}$, photographs of plates were taken and densitometric measures of the growth of strains were performed using the ImageJ software package (Schneider et al., 2012). Susceptibility of strains was determined by comparison of growth on selective plates relative to control plates for two biological replicates.

\section{Outer membrane protein analysis}

After $15 \mathrm{~h}$ growth overnight in a chemostat containing $0.1 \times$ M9 supplemented with $4 \%$ (vol/vol) LSLB, $80 \mathrm{ml}$ of bacterial culture was harvested by centrifugation 5000 r.p.m. for $10 \mathrm{~min}$ and resuspended in $0.2 \mathrm{ml}$ sterile water. Bacteria were boiled for $10 \mathrm{~min}$ at $100{ }^{\circ} \mathrm{C}$ before centrifugation at 12000 r.p.m. for $5 \mathrm{~min}$ and resuspended in $0.2 \mathrm{ml}$ sample buffer $(50 \mathrm{~mm}$ Tris-HCl, $4 \%$ SDS, $16 \%$ glycerol, $0.0048 \%$ bromophenol blue, $4.8 \% \beta$-mercaptoethanol). Samples were boiled for $10 \mathrm{~min}$ and chilled on ice for $5 \mathrm{~min}$. After centrifugation for $10 \mathrm{~min}$ at $4{ }^{\circ} \mathrm{C}$, the supernatant was analysed by SDS-polyacrylamide gel electrophoresis in the presence of urea (Liu and Ferenci, 1998). Proportions of outer membrane protein bands were determined using the densitometric function of ImageJ (Schneider et al., 2012).

Outer membrane permeability assay

Strains to be tested were electroporated with the plasmid pBR322 encoding high levels of $\beta$-lactamase. Outer membrane permeability was measured by comparing $\beta$-lactamase activity in whole cells (with intact outer membrane) with that in disrupted cells with no barrier, using the permeable colorimetric substrate nitrocefin (Oxoid, Addaide, SA, Australia; Uri, 1985). Bacteria were grown to exponential phase at $37^{\circ} \mathrm{C}$ in LSLB containing $100 \mu \mathrm{g} \mathrm{ml}^{-1}$ ampicillin and $15 \mu \mathrm{g} \mathrm{ml}^{-1}$ tetracycline. The optical density of the culture was measured and two $100 \mu \mathrm{l}$ samples were taken. One aliquot (whole cells) was washed once, resuspended in the same volume of $0.1 \times \mathrm{M} 9$ and the second aliquot was centrifuged and resuspended in $30 \%$ sucrose, $33 \mathrm{~mm}$ Tris, $5 \mu \mathrm{l} 2 \mathrm{mg} \mathrm{ml}^{-1}$ lysozyme and $5 \mu \mathrm{l} 0.2 \mathrm{M}$ EDTA, and the mixture placed on ice for $15 \mathrm{~min}$. Lysed bacteria were centrifuged for $5 \mathrm{~min}$ at $4{ }^{\circ} \mathrm{C}$, the pellet discarded and $3 \mu \mathrm{l} 1 \mathrm{M} \mathrm{MgCl}_{2}$ added to the supernatant. The broken cells and whole cells were assayed in 96-well microtitre plates with each well containing $160 \mu \mathrm{l} 0.1 \times \mathrm{M} 9,20 \mu \mathrm{l} 50 \mathrm{mg} \mathrm{ml}^{-1}$ nitrocefin and $20 \mu \mathrm{l}$ of the sample. The 96-well plate reader (Multiskan RC plate reader Labsystems Oy, Helsinki, Finland) was configured for kinetics at $492 \mathrm{~nm}$ for $20 \mathrm{~min}$, with readings taken every $2 \mathrm{~min}$. The activity is presented as the rate of optical density change per minute per cell in the assay.

\section{Sequence analysis}

PCR amplification of the ompC gene used the two external primers, C1 (5'-AAGGTACTGTCCCTC CTGG-3') and C3 (5'-GCAGGCCCTTTGTTCGAT-3'; Zhang and Ferenci, 1999). PCR amplification of the $o m p F$ gene of ECOR59 and ECOR59E used two primers: OmpFF1 (5'-TGCTCTGTTAGTAGC-3') and OmpFR1 (5'-GATTGCTCTGGAAGG- $\left.3^{\prime}\right)$. The PCR product was purified using the Wizard PCR preps DNA purification system (Promega Corp., Madison, WI, USA). The nucleotide sequence was determined using the above primers and dye-terminator sequencing reactions at the Australian Genome Research Facility (Sydney node, NSW, Australia). Mutations in the $o m p F$ sequence were detected by alignment between sequences using Bioedit software (downloaded from: (http://www.mbio.ncsu.edu/bioedit/). The OmpF structure (Cowan et al., 1995) was obtained from the Protein Data Bank and the position of mutations were visualized using PyMOL (downloaded from: http://www.pymol.org/

\section{Results}

Competitive fitness in members of the species E. coli The ability of bacteria to compete for nutrients is best measured in a chemostat system where the 
environmental parameters are under continuous control. To study species-wide competitiveness, we used MG1655 as a reference; this is a much used laboratory K-12 strain of E. coli (Hayashi et al., 2006). In Figure 2, we investigated fitness with mixtures of substrates (either LSLB or mixtures of sugars) as limiting nutrients in chemostats. The use of mixtures was to ensure that the measure of fitness was more dependent on porin-dependent general permeability with all polar substrates than on the use of a single sugar or amino acid. The diluted rich medium, LSLB broth, with mainly amino acids as nutrients, was the most intensively investigated (Figure 2a). A mixture of four sugars was also used in minimal media as limiting carbon source in a subset of strains (Figure 2b). Under nutrient limitation, mixtures of substrates are used simultaneously (Lendenmann et al., 1996). After mixing chemostatacclimatized MG1655 and individual ECOR strains in a 50:50 ratio, competition over 2 days was used to measure changes in sub-populations, calculated as a selection coefficient $\boldsymbol{s}$ (Dykhuizen and Hartl, 1983). As indicated in Figure 2, the ECOR collection exhibited a continuum of fitness characteristics in both types of media, with some strains being much fitter or less fit than the reference strain. The $\boldsymbol{s}$ value of ECOR strains in $0.1 \times$ M9 supplemented with LSLB or with mixed sugars when compared using Spearman's rank order correlation showed a strong
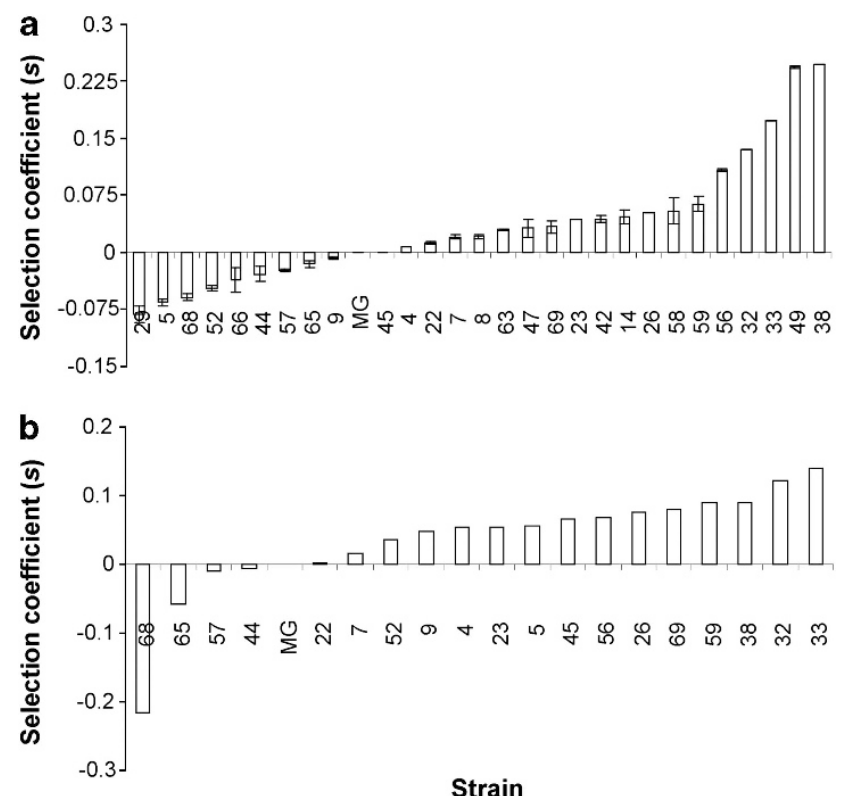

Figure 2 The competition of ECOR strains for nutrients under nutrient limitation. (a) Competition against the MG1655 reference strain was in chemostats fed with media containing $0.1 \times$ M9+4\% LSLB. The s.d. of two independent competitions with two replicates are shown. (b) Competition was in single chemostats fed with media containing $0.1 \times \mathrm{M} 9+$ with $0.005 \%$ glucose, $0.005 \%$ galactose, $0.005 \%$ fructose and $0.005 \%$ xylose. A single competition was performed. The ECOR strain number is shown below each result; strains with a negative value are less fit than MG1655 (MG) whereas the positive values indicate higher fitness. positive correlation in fitness trends between the two conditions $(r=0.735294, P=0.0032)$. Some variation between sugars and LSLB was observed for individual ECOR strains, probably owing to polymorphisms in amino acid or sugar metabolism. Nevertheless, the strong correlation in fitness trends between the two media suggests that the observed differences are caused by common organismic properties.

Diversity in susceptibility to antibacterial agents in the species E. coli

The hypothesis we wished to test was whether the competitive ability of strains was related to the permeability of bacteria at the level of the outer membrane. An indirect, frequently used test for outer membrane permeability is susceptibility to antibiotics and detergents, as outer membrane exclusion is an important component of protection against these compounds (Liu and Ferenci, 2001; Nikaido, 2003; Pages et al., 2008; Delcour, 2009). We thus tested the susceptibility of the ECOR set to low but inhibitory concentrations of a detergent (SDS) and a polar antibiotic $(\mathrm{Cml})$ that diffuses through both OmpF and OmpC porin channels (Mortimer and Piddok, 1993). As shown in Figure 3a, growth on plates containing these antibacterial compounds was highly heterogeneous in the ECOR set. Using a densitometric measurement of growth on such replicate plates, Figure $3 \mathrm{~b}$ shows a more quantitative comparison of the effects of SDS and Cml; as can be seen there is a continuum of susceptibilities to $\mathrm{Cml}$ across the species, whereas the SDS has a more bimodal distribution amongst susceptible and more resistant strains at the concentration used. Previous surveys that have shown that ECOR37 is the only strain that exhibits clinical levels of resistance to Cml (Houndt and Ochman, 2000; Mazel et al., 2000); this is evident in Figure 3b. Hence, the ECOR representation of species $E$. coli exhibits a considerable diversity in antibacterial susceptibilities. Importantly, there was a strong correlation between the susceptibilities to the two different compounds when compared using Spearman's rank order correlation ( $r=0.5578, P=<0.000001)$, pointing to a common component in susceptibilities.

\section{A trade-off between competitive ability and susceptibility to antibacterial agents in the species E. coli}

To test the pattern of a relationship between fitness and antibacterial susceptibilities, Figure 4 shows a plot of $s$ values of each strain against its ability to grow in the presence of antibacterials. For clarity, Figure 4 does not show the individual ECOR identities of the points, but Supplementary Table S1 contains the data. Evidently, the highest competitive ability for nutrients is always linked with high susceptibility (low growth) with either SDS or Cml. 


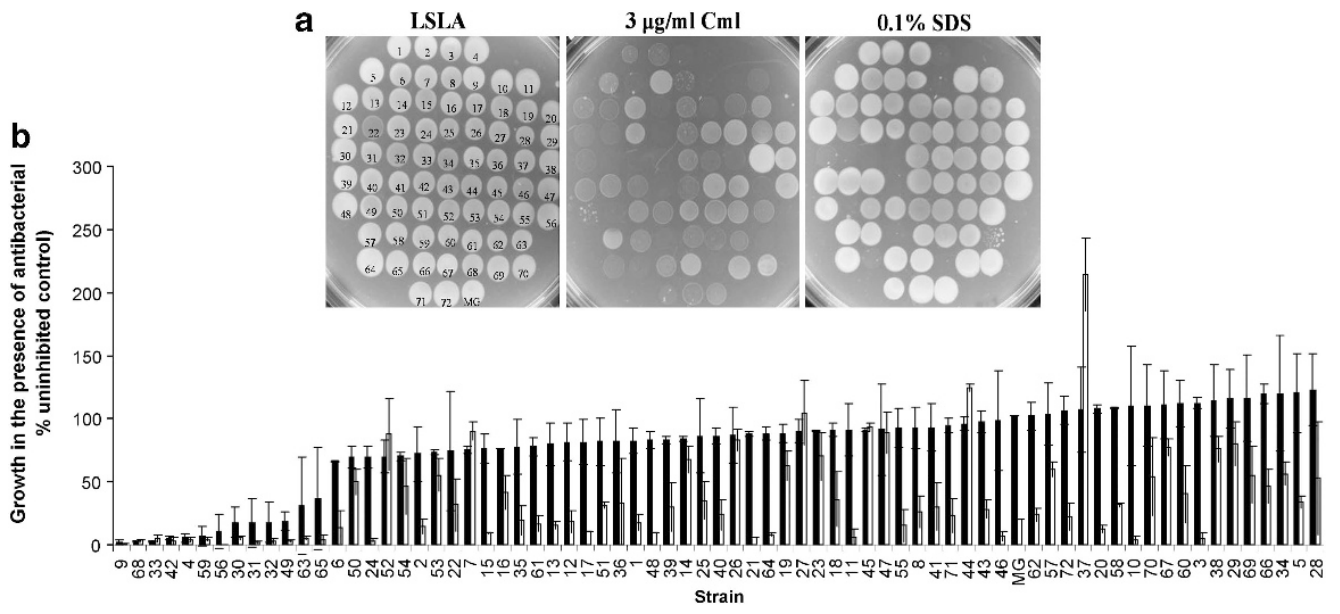

Figure 3 Antibiotic and detergent susceptibility of ECOR strains. (a) Growth on spot plates of the ECOR strains is shown on LSL-agar (LSLA, uninhibited control) and on LSLA $+3 \mu \mathrm{g} \mathrm{ml}{ }^{-1} \mathrm{Cml}$ and LSLA $+0.1 \%$ SDS. (b) The results of densitometric analysis of growth on LSLA $+3 \mu \mathrm{g} \mathrm{ml}^{-1} \mathrm{Cml}$ (open bars) and LSLA + 0.1\% SDS (filled bars) relative to growth on control plates from two biological replicates.

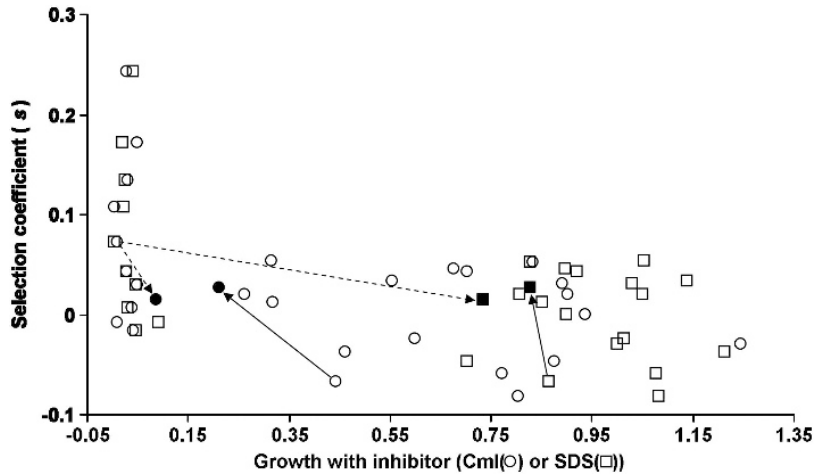

Figure 4 The trade-off between fitness under nutrient limitation and resistance to antibacterial compounds. Selection coefficients were measured in chemostats fed with media containing $0.1 \times$ $\mathrm{M} 9+4 \%$ LSLB. Growth in the presence of $3 \mu \mathrm{g} \mathrm{ml}{ }^{-1} \mathrm{Cml}$ (circles) or $0.1 \%$ SDS (squares) were quantitated on spot plates as in Figure 3. The arrows link the altered positions in the trade-off of ECOR strains evolved as described in the text, from ECOR59 (dashed arrows) to ECOR59E (filled symbols) and ECOR5 (solid arrows) to ECOR5E (filled symbols).

Also, all the more resistant organisms were less competitive. These results are indicative of a tradeoff, but the shape of the trade-off is difficult to fit with high correlation coefficients in curve-fitting programs. If, as seems likely, a strongly convex shape describes the fitness/susceptibility relationship, the theoretical consequence of this form is likely to be the evolution of generalists, and most natural E. coli are indeed between the extremes of resistance and sensitivity.

Shifting the setting of the trade-off between competitive ability and susceptibility to antibacterial agents A trade-off with naturally different settings as shown in Figure 4 should reassort when a generalist is subject to a selection in a new, suboptimal environment. To test whether the trade-off between susceptibility and $\boldsymbol{s}$ is indeed linked to alterations in
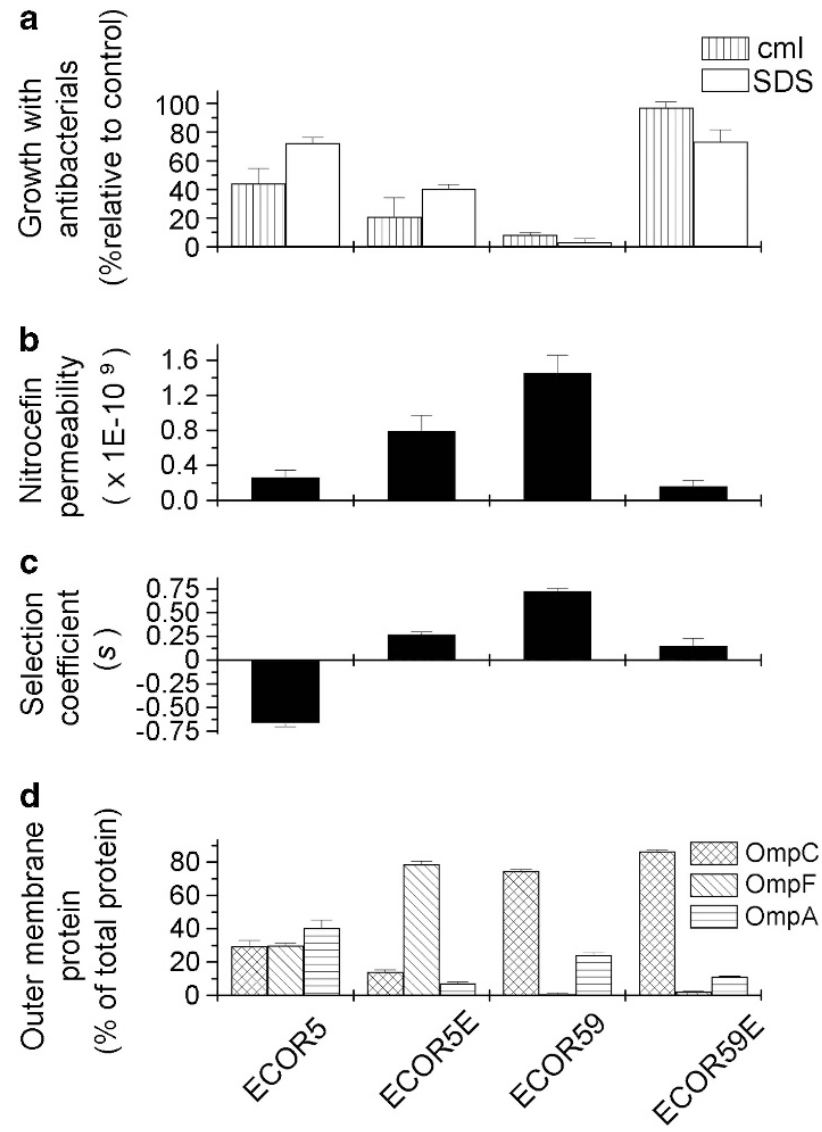

Figure 5 The phenotypes of strains evolved from ECOR5 and ECOR59. (a) Growth on selective plates containing Cml (stripes) and SDS (white). The concentrations used were $3 \mu \mathrm{g} \mathrm{ml}^{-1}$ and $1 \mu \mathrm{g} \mathrm{ml}^{-1} \mathrm{Cml}$ for strains ECOR5/ECOR5E and ECOR59/ ECOR59E, respectively, and $0.1 \%$ SDS and $5 \%$ SDS for ECOR59/ECOR59E and ECOR5/ECOR5E, respectively. (b) Permeability was measured in LSLB batch culture by the rate of colour change after the addition of the colorometric $\beta$-lactam nitrocefin (24 replicates). (c) Selection coefficients were measured in four independent chemostats for each strain fed with media containing $0.1 \times \mathrm{M} 9+4 \%$ LSLB. (d) Outer membrane porin content. OmpC (mottled), OmpF (diagonals) and OmpA (horizontal stripes) were estimated from three independent experiments. 
outer membrane permeability, as the above results suggest, we took a more sensitive (ECOR59) and a more resistant (ECOR5) strain and subjected them to selection in an environment likely to shift the setting of the trade-off. ECOR59 was made more resistant by selection on a $1 \mu \mathrm{gml}^{-1} \mathrm{Cml}$ plate, picking colonies able to grow better. ECOR5 was selected to grow well in chemostats limited by LSLB. After 10 days in the chemostat, a mutational sweep was evident because $72 \%$ of isolates were more sensitive to SDS than ECOR5. Colonies obtained after both selections were then screened for the phenotypes shown in Figure 5, in each case in comparison with the original ECOR strains.

As shown by an example of an adapted strain derived from each ECOR ancestor (ECOR5E derived from ECOR5 and ECOR59E from ECOR59), the sensitivity to antibacterials and competitive fitness both changed (Figure 5a). The fitness of each evolved strain also changed reciprocally to the shift in susceptibility (Figure 5c). Importantly, susceptibility and fitness changed even when either was the unselected trait; as shown in Figure 4, the position of each adapted strain moved in the trade-off curve in line with a reciprocal relationship between antibacterial sensitivity and competitive fitness. Coincidentally, the evolved clones converged to become very similar in their fitness as well as in assayed resistance to SDS and Cml (Figure 4).

The molecular changes in the adapted mutants were investigated, focusing on functional or regulatory changes in outer membrane permeability and porin proteins. Regulatory changes in porin expression were detected using SDS-urea polyacrylamide electrophoresis and the examination of the three major outer membrane proteins, including the two porins $\mathrm{OmpF}$ and $\mathrm{OmpC}$, and the structural protein
OmpA (Nikaido, 2003). As displayed in Figure 5d, the expression of the OmpF band was increased from $27 \%$ of major protein expression in ECOR 5 to $70 \%$ in ECOR5E, as measured by densitometry. As OmpF forms the larger channel, increased levels of $\mathrm{OmpF}$ can explain the increase in outer membrane permeability in ECOR5E. The mutational source of the regulatory change was not identified, but could be potentially affect any of the numerous inputs into $o m p F$ and ompC regulation (Liu and Ferenci, 2001).

The change in outer membrane protein levels in ECOR59E were much less pronounced than in ECOR5E, so to detect possible structural changes in porins, both the ompF and ompC genes were sequenced. No changes were found in ECOR5E, but ECOR59E exhibited ompF changes resulting in four amino-acid substitutions. As illustrated in Figure 6, one mutation resulted in a non-conservative substitution affecting position 103 in the channelconstricting L3 loop of OmpF. In addition, two of the three substitutions later in the sequence resulted in changes to residues 331 and 333 that point into the $\beta$-barrel structure (Figure 6). The combination of these changes could readily explain reduced permeability to antibacterials and nutrients.

To show the effects of the adaptations in ECOR5E and ECOR59E on permeability directly, outer membrane diffusion rates were measured from the permeation of nitrocefin, a $\beta$-lactam, which is hydrolysed by $\beta$-lactamase to a coloured product after crossing into the periplasm. All strains subject to this assay were transformed by a plasmid encoding periplasmic $\beta$-lactamase. As shown in Figure $5 \mathrm{~b}$, the rate of diffusion across the outer membrane was well correlated with selection coefficients and inversely with antibacterial susceptibility. In total, the results with the two

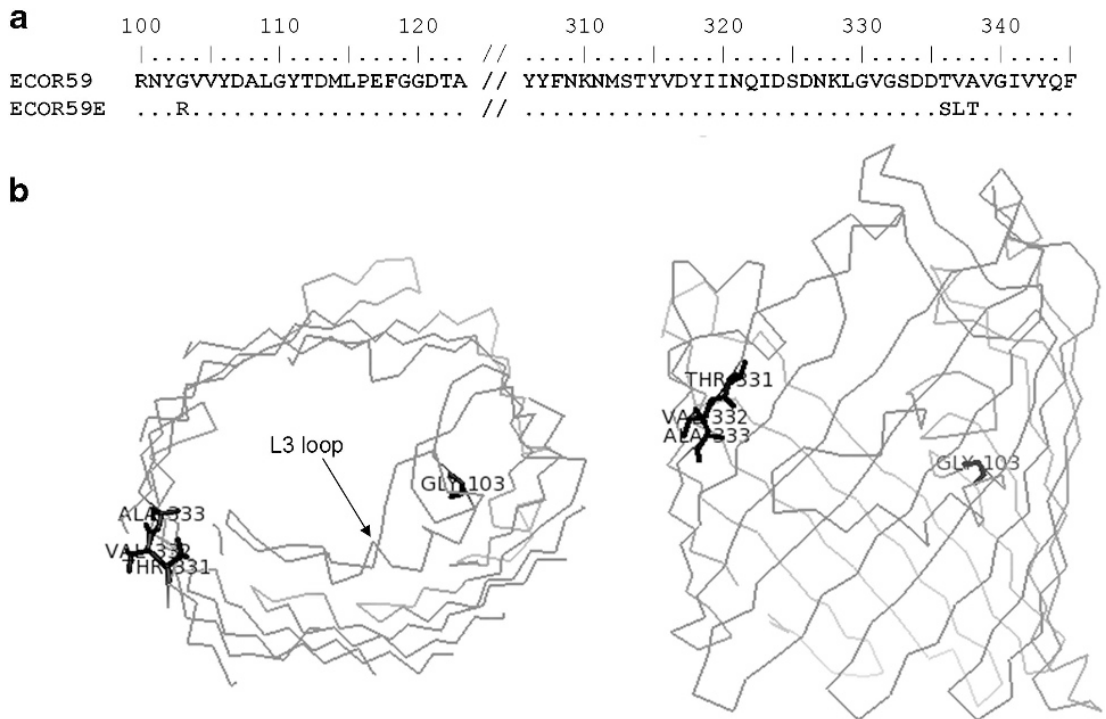

Figure 6 Substitutions in the ECOR5E OmpF porin channel. (a) Mutations causing amino-acid substitutions in the OmpF sequence. (b) The backbone of a monomeric OmpF porin from the Protein Data Bank (2OMF) with the L3 channel constriction loop and the changes in ECOR5E (in bold type, showing side chains). 
environmentally adapted ECOR strains are consistent with changes in membrane permeability shifting the setting of the nutrition-resistance trade-off in E. coli.

\section{Discussion}

The capacity to compete for nutrient with high affinity has long been considered an important ecological adaptation in free-living bacteria that are mostly subject to environmental limitation for nutrients (Poindexter, 1981; Button, 1998). Tabulations of nutrient affinities (Button, 1998) and mechanistic studies have indeed pointed to the presence of high-affinity transport systems in bacteria (Ferenci, 1996). The underlying assumption has been that nutrient affinities have evolved to enhance the competitive fitness of bacteria in lowsubstrate conditions that they often live in, generally in competition with other species. E. coli certainly needs to compete with other organisms both in the highly populated colon and in secondary environments (Tenaillon et al., 2010). Perhaps surprisingly, our data suggest that affinity for nutrients is not optimized in most isolates. We present evidence that scavenging ability is subject to trade-offs that limit competitive ability in individuals, but not necessarily in the species as a whole. The extensive diversity in competitive characteristics we find under nutrient limitation within a bacterial species suggests that high affinity carries a cost.

The ECOR strains are ecologically diverse coming from different animal hosts, some from infections and with a global source distribution (Ochman and Selander, 1984). The phylogenetic diversity within the species E. coli and the ECOR collection has been extensively demonstrated (Wirth et al., 2006). We found no clear correlations between the ecological source of ECOR strains (Ochman and Selander, 1984), their phylogenetic relationships (Wirth et al., 2006) and their position in the competitionresistance trade-off. For example, the fittest strains ECOR32, 33 and 38 came from a giraffe, a sheep and a human, respectively, whereas the less fit strains (ECOR68 and ECOR5) also included giraffe and human isolates, respectively. The heterogeneity we see is most likely a microevolutionary response to nutrient- or antibacterial stress and presumably happens commonly in the situations that E. coli meets in nature. The conflicting environmental demands on fitness mean that there is a significant proportion of E. Coli isolates that evolved to compromise and have an intermediate resistance to antibacterials rather than optimum nutrient permeability. This pattern, together with the possibility for mutational change, offers the advantage of bethedging options, some strains being competitive, some resistant, many with a range of intermediate properties (Ryall et al., 2012). This is understandable because E. coli is frequently exposed to detergents in the intestinal tract and resistance to bile salts is also porin dependent (De Paepe et al., 2011). The broad continuum of phenotypes presented by the ECOR set also suggests that the SPANC trade-off is frequently reassorted in nature, giving multiple resistance-competitiveness settings in the species. In addition, starting from any point in the trade-off curve, mutational selection can reset the SPANC balance, in either the resistance or nutritional competitiveness directions.

The mutational and physiological changes in porins and outer membrane permeability in ECOR strains selected to move along the trade-off curve point to the mechanistic basis of the trade-off. The outer membrane permeability assays of ECOR strains and their evolved derivatives are entirely consistent with permeability changing with altered settings of the trade-off. Changes either in the amount of porin or the structure of the porin can result in an altered competition-resistance trade-off. Permeability is determined by the proportion of OmpF and OmpC proteins expressed; the ECOR5E strain with increased competitive ability has a major shift towards the expression of $\mathrm{OmpF}$, the largechannel porin. In the ECOR59E strain with reduced susceptibility to antibiotic, the permeability of the outer membrane was reduced by a multi-residue structural change in amino acids lining the channel in the OmpF protein. These results are consistent with observations that altered porins, or their regulation are often responsible for cefoxitin resistance in E coli (Clarke et al., 2003). Conversely, when porins are forced to permeate larger di- or trisaccharides as growth substrates (Misra and Benson, 1988; Zhang and Ferenci, 1999), mutations in porin regulation and structure can overcome the permeability limitation, but always resulted in increased sensitivity to antibiotic and detergent (Misra and Benson, 1988; Zhang and Ferenci, 1999).

The trade-off curve shown in Figure 4 separates areas of trait space that contain and do not contain feasible phenotypes. The markedly nonlinear shape of the trade-off indicates that elevation of competitiveness requires high susceptibility to antibacterial agents and high membrane permeability. The geometry of the trade-off also has important evolutionary consequences (Levins, 1968; de Mazancourt and Dieckmann, 2004) and if a convex shape as in Figure 4 is found, a tendency to evolve into a generalist is favoured in the evolution of the phenotypes (Levins, 1968). Indeed, the majority of the ECOR strains are not at the extremes of stress resistance or nutritional competitiveness, so most E. coli isolates are at least part generalists, accommodating intermediate levels of stress and competitiveness.

A previously reached conclusion is that E. coli isolates all have similar fitness and growth characteristics (Ihssen et al., 2007). This conclusion is not at all borne out by our far more extensive comparisons. The earlier studies were based on a 
small number of isolates from Swiss aquatic environments and it is possible that these isolates have already converged on a particular growth stress trade-off. In contrast, the ECOR strains are from a variety of animal sources and geographical locations (Selander et al., 1987), and are thus more representative of the diversity within the E. coli species. Figure 2 indicates that there is, species-wide, a very broad distribution of fitness capabilities and there is no species typical fitness or stress resistance, as suggested previously (Ihssen et al., 2007).

Studies of trade-offs in E. coli are beginning to reveal that the SPANC balance, and probably survival multiplication trade-offs generally, are the sum of several mechanistic contributions. The tradeoff in resource allocation between vegetative and stress genes involving RpoS has been previously explored (King et al., 2004). There is a species-wide variation in RpoS levels, making some cells more resistant to environmental stresses such as low $\mathrm{pH}$, cold, oxidative stress and high osmolarity (high RpoS strains), but these cells also have limited competitive ability in nutrient-poor conditions. Other, nutritionally competitive strains in nature have low or no RpoS-dependent stress response (King and Ferenci, 2005; Ferenci et al., 2011). The RpoS levels within the ECOR set have been examined (Ferenci et al., 2011), so it is now possible to compare the influence of RpoS level on the susceptibility with antibiotics as well as the competitive ability (s) of strains. As shown in Supplementary Figure S1a, there is no clear linkage between RpoS levels and susceptibility to either SDS or $\mathrm{Cml}$. Likewise, as shown in Supplementary Figure S1b, there is a noisy relationship, with no obvious trends, in plotting RpoS levels against competitive fitness. The extensive diversity in global regulation as well as other polymorphisms between natural isolates, as was demonstrated for ppGpp levels (Ferenci et al., 2011), makes it more difficult to define a specieswide trade-off involving RpoS.

RpoS levels have also been shown to affect porin levels within K-12 strains of $E$. coli, because the global transcriptional effects of RpoS include changes in the proportion of $\mathrm{OmpF}$ and $\mathrm{OmpC}$ porins, with high RpoS having a negative effect on OmpF levels in $E$. coli K-12 (Liu and Ferenci, 2001). High RpoS strains are thus more expected to exclude antibacterials. However, comparison of RpoS levels to growth on antibacterials (Supplementary Figure S1a) showed little correlation between the two traits; some strains with low RpoS were highly resistant to antibiotics. Furthermore, the regulatory change in porin levels in ECOR5E was not due to a change in RpoS function, so other regulatory heterogeneities affecting the complex regulation of porins may also affect $E$. coli (Pratt et al., 1996; Liu and Ferenci, 2001). As such, despite the links between RpoS and porin levels in laboratory K-12 strains, we found no convincing evidence of an overlap between the RpoS- and outer membrane-based trade-offs.
There is a message from our results beyond the ecological, mechanistic and diversity-related conclusions. Our results clearly show the considerable cost of reduced antibiotic susceptibility is the reduced competitiveness. This raises the question of whether the changes of outer membrane permeability commonly found in clinical isolates exhibiting drug exclusion carry a cost to the resistant organism. A systematic study of fitness costs of reduced antibiotic permeability is needed in clinical isolates; the ECOR set is mainly a collection of commensal strains, so we cannot readily extrapolate to clinical isolates. There is some evidence that permeability changes and diversifies in patients (Levert et al., 2010), but the competitive costs of these changes has not been evaluated. A more detailed study is needed with accurate competition experiments as used in this study. The prediction arising from our results is that many clinical isolates will be less competitive against less resistant strains, unless compensatory mutations improving fitness occur (Tanaka and Valckenborgh, 2011).

\section{Conflict of Interest}

The authors declare no conflict of interest.

\section{Acknowledgements}

We thank Ram Maharjan for comments on the manuscript and the Australian Research Council for grant support.

\section{References}

Agrawal AA, Conner JK, Rasmann S. (2010). Tradeoffs and negative correlations in evolutionary ecology. In: WFE Bell MA, Futuyma DJ, Levinton JS (eds) Evolution After Darwin: the First 150 Years. Sinauer Associates: Sunderland, MA, pp 243-268.

Bavoil P, Nikaido H, von Meyenburg K. (1977). Pleiotropic transport mutants of Escherichia coli lack porin, a major outer membrane protein. Mol Gen Genet 158: 23-33.

Button DK. (1998). Nutrient uptake by microorganisms according to kinetic parameters from theory as related to cytoarchitecture. Microbiol Mol Biol Rev 62: 636-645.

Clarke B, Hiltz M, Musgrave H, Forward KR. (2003). Cephamycin resistance in clinical isolates and labotatory-derived strains of Escherichia coli, Nova Scotia, Canada. Emerging Infect Dis 9: 1254-1259.

Cooper VS, Lenski RE. (2000). The population genetics of ecological specialization in evolving Escherichia coli populations. Nature 407: 736-739.

Cowan SW, Garavito RM, Jansonius JN, Jenkins JA, Karlsson R, Konig N et al. (1995). The structure of OmpF porin in a tetragonal crystal form. Structure 3: 1041-1050.

de Mazancourt C, Dieckmann U. (2004). Trade-off geometries and frequency-dependent selection. Am Nat 164: 765-778. 
De Paepe M, Taddei F. (2006). Viruses' life history: towards a mechanistic basis of a trade-off between survival and reproduction among phages. PLoS Biol 4: e193.

De Paepe M, Gaboriau-Routhiau V, Rainteau D, Rakotobe S, Taddei F, Cerf-Bensussan N. (2011). Trade-off between bile resistance and nutritional competence drives Escherichia coli diversification in the mouse gut. PLoS Genet 7: 2107-2107.

Delcour AH. (2009). Outer membrane permeability and antibiotic resistance. Biochim Biophys Acta 1794: 808-816.

Dykhuizen DE, Hartl DE. (1983). Selection in chemostats. Microbiol Rev 47: 150-168.

Ferenci T. (1996). Adaptation to life at micromolar nutrient levels: the regulation of Escherichia coli glucose transport by endoinduction and cAMP. FEMS Microbiol Rev 18: 301-317.

Ferenci T. (2005). Maintaining a healthy SPANC balance through regulatory and mutational adaptation. Mol Microbiol 57: 1-8.

Ferenci T, Galbiati H, Betteridge T, Phan K, Spira B. (2011). The constancy of global regulation across a species: the concentrations of ppGpp and RpoS are strain-specific in Escherichia coli. BMC Microbiol 11: 62.

Giaever HM, Styrvold OB, Kaasen I, Strom AR. (1988). Biochemical and genetic characterisation of osmoregulatory trehalose synthesis in Escherichia coli. J Bacteriol 170: 2841-2849.

Gudelj I, Weitz JS, Ferenci T, Horner-Devine MC, Marx CJ, Meyer JR et al. (2010). An integrative approach to understanding microbial diversity: from intracellular mechanisms to community structure. Ecol Lett 13: 1073-1084.

Hayashi K, Morooka N, Yamamoto Y, Fujita K, Isono K, Choi $S$ et al. (2006). Highly accurate genome sequences of Escherichia coli K-12 strains MG1655 and W3110. Mol Syst Biol U95-U99.

Hengge R. (2011). The General Stress Response in GramNegative Bacteria. ASM Press: Washington DC, USA, pp 251-289.

Houndt T, Ochman H. (2000). Long-term shifts in patterns of antibiotic resistance in enteric bacteria. Appl Env Microbiol 66: 5406-5409.

Ihssen J, Grasselli E, Bassin C, Francois P, Piffaretti JC, Koster W et al. (2007). Comparative genomic hybridization and physiological characterization of environmental isolates indicate that significant (eco-) physiological properties are highly conserved in the species Escherichia coli. Microbiology (SGM) 153: 2052-2066.

Jessup CM, Bohannan BJM. (2008). The shape of an ecological trade-off varies with environment. Ecol Lett 11: 947-959.

Kang YS, Park W. (2010). Trade-off between antibiotic resistance and biological fitness in Acinetobacter sp strain DR1. Env Microbiol 12: 1304-1318.

King T, Ishihama A, Kori A, Ferenci T. (2004). A regulatory trade-off as a source of strain variation in the species Escherichia coli. J Bacteriol 186: 5614-5620.

King T, Ferenci T. (2005). Divergent roles of RpoS in Escherichia coli under aerobic and anaerobic conditions. FEMS Microbiol Lett 244: 323-327.

King T, Seeto S, Ferenci T. (2006). Genotype-by-environment interactions influencing the emergence of rpoS mutations in Eschetichia coli populations. Genetics 172: 2071-2079.

Lang GI, Murray AW, Botstein D. (2009). The cost of gene expression underlies a fitness trade-off in yeast. Proc Natl Acad Sci USA 106: 5755-5760.

Lee MC, Chou HH, Marx CJ. (2009). Assymetric, bimodal trade-offs during adaptation of Methylobacterium to distinct growth substrates. Evolution 63: 2816-2830.

Lendenmann U, Snozzi M, Egli T. (1996). Kinetics of the simultaneous utilization of sugar mixtures by Escherichia coli in continuous culture. Appl Env Microbiol 62: 1493-1499.

Levert M, Zamfir O, Clermont O, Bouvet O, Lespinats S, Hipeaux MC et al. (2010). Molecular and evolutionary bases of within-patient genotypic and phenotypic diversity in escherichia coli extraintestinal infections - art. no. e1001125. PLoS Path 6: 1125-1125.

Levins R. (1968). Evolution in changing environments; some theoretical exploration. Princeton University Press: Princeton, New Jersey, USA.

Liu XQ, Ferenci T. (1998). Regulation of porin-mediated outer membrane permeability by nutrient limitation in Escherichia coli. J Bacteriol 180: 3917-3922.

Liu XQ, Ferenci T. (2001). An analysis of multifactorial influences on the transcriptional control of ompF and ompC porin expression under nutrient limitation. Microbiology 147: 2981-2989.

MacLean RC, Bell G, Rainey PB. (2004). The evolution of a pleiotropic fitness tradeoff in Pseudomonas fluorescens. Proc Natl Acad Sci USA 101: 8072-8077.

Maharjan R, Seeto S, Notley-McRobb L, Ferenci T. (2006). Clonal adaptive radiation in a constant environment. Science 313: 514-517.

Maharjan R, McKenzie C, Yeung A, Ferenci T. (2013). The basis of antagonistic pleiotropy in $h f q$ mutations that have opposite effects on fitness at slow and fast growth rates. Heredity 110: 10-18.

Mazel D, Dychinco B, Webb VA, Davies J. (2000). Antibiotic resistance in the ECOR collection: integrons and identification of a novel AAD gene. Antimicrob Agents Chemother 44: 1568-1574.

Miller J. (1972). Experiments in molecular genetics. Cold Spring Harbor Laboratory: Cold Spring Harbor, NY.

Misra R, Benson SA. (1988). Isolation and characterization of OmpC porin mutants with altered pore properties. J Bacteriol 170: 528-533.

Mortimer PGS, Piddok LJV. (1993). The accumulation of five antibacterial agents in porin-deficient mutants of Escherichia coli. I Antimicrob Chemother 32: 195-213.

Mroczkowska JE, Barlow M. (2008). Fitness trade-offs in bla(TEM) evolution. Antimicrob Agents Chemother 52: 2340-2345.

Nikaido H. (2003). Molecular basis of bacterial outer membrane permeability revisited. Microbiol Mol Biol Rev 67: 593-656.

Notley-McRobb L, King T, Ferenci T. (2002). rpoS mutations and loss of general stress resistance in Escherichia coli populations as a consequence of conflict between competing stress responses. J Bacteriol 184: 806-811.

Nystrom T. (2004). Growth versus maintenance: a trade-off dictated by RNA polymerase availability and sigma factor competition? Mol Microbiol 54: 855-862.

Ochman H, Selander RK. (1984). Standard reference strains of Escherichia coli from natural populations. J Bacteriol 157: 690-693. 
Pages JM, James CE, Winterhalter M. (2008). The porin and the permeating antibiotic: a selective diffusion barrier in Gram-negative bacteria. Nat Rev Microbiol 6: 893-903.

Poindexter JS. (1981). Oligotrophy: fast and famine existence. Adv Microb Ecol 5: 63-89.

Pratt LA, Hsing WH, Gibson KE, Silhavy TJ. (1996). From acids to OsmZ -multiple factors influence the synthesis of the $\mathrm{OmpF}$ and $\mathrm{OmpC}$ porins in Escherichia coli. Mol Microbiol 20: 911-917.

Pupo GM, Lan RT, Reeves PR, Baverstock PR. (2000). Population genetics of Escherichia coli in a natural population of native Australian rats. Env Microbiol 2: 594-610.

Roff DA, Fairbairn DJ. (2007). The evolution of trade-offs: where are we? J Evol Biol 20: 433-447.

Ryall B, Eydallin G, Ferenci T. (2012). Culture history and population heterogeneity as determinants of bacterial adaptation: the adaptomics of a single environmental transition. Microbiol Mol Biol Rev 76: 597-625.

Schneider CA, Rasband WS, Eliceiri KW. (2012). NIH Image to ImageJ: 25 years of image analysis. Nat Methods 9: 671-675.

Selander RK, Caugant DA, Whittam TS. (1987). Genetic structure and variation in natural populations of Escherichia coli. In: Neidhardt FC (eds) Escherichia coli and Salmonella typhimurium. Cellular and molecular biology, 1st edn, ASM Press: Washington DC, pp 1625-1648.

Stearns SC. (1989). Trade-offs in life-history evolution. Funct Ecol 3: 259-268.

Supplementary Information accompanies this paper on The ISME Journal website (http://www.nature.com/ismej)
Tanaka MM, Valckenborgh F. (2011). Escaping an evolutionary lobster trap: drug resistance and compensatory mutation in a fluctuating environment. Evolution 65: 1376-1387.

Taylor TB, Buckling A. (2011). Selection experiments reveal trade-offs between swimming and twitching motilities in Pseudomonas aeruginosa. Evolution 65: 3060-3069.

Tenaillon O, Skurnik D, Picard B, Denamur E. (2010). The population genetics of commensal Escherichia coli. Nat Rev Microbiol 8: 207-217.

Touchon M, Hoede C, Tenaillon O, Barbe V, Baeriswyl S, Bidet $\mathrm{P}$ et al. (2009). Organised genome dynamics in the Escherichia coli species results in highly diverse adaptive paths. PLoS Genet 5: e1000344.

Tree JJ, Ulett GC, Ong CLY, Trott DJ, McEwan AG, Schembri MA. (2008). Trade-off between iron uptake and protection against oxidative stress: deletion of cueO promotes uropathogenic Escherichia coli virulence in a mouse model of urinary tract infection. J Bacteriol 190: 6909-6912.

Uri JV. (1985). Detection of beta-lactamase activity with nitrocefin of multiple strains of various microbial genera. Acta Microbiologica Hungarica 32: 133-145.

Wirth T, Falush D, Lan RT, Colles F, Mensa P, Wieler LH et al. (2006). Sex and virulence in Escherichia coli: an evolutionary perspective. Mol Microbiol 60: 1136-1151.

Zhang E, Ferenci T. (1999). OmpF changes and the complexity of Escherichia coli adaptation to prolonged lactose limitation. FEMS Microbiol Lett 176: 395-401. 